\title{
471 UNDERSTANDING VARIATION IN SUCCESS IN A QUALITY IMPROVEMENT INITIATIVE IN SASKATCHEWAN, CANADA.
}

Gary Groot, ${ }^{1}$ Linda McMullen, ${ }^{1}$ Jessica Hamilton, ${ }^{2}$ Laura Schwartz ${ }^{2} .{ }^{1}$ University of Saskatchewan, Canada; ${ }^{2}$ Saskatchewan Health Quality Council, Canada

10.1136/bmjqs-2015-IHlabstracts.5

Background Unexplained variation in clinical practice can be an indicator of poor quality of care. In Saskatchewan, Canada the Variation and Appropriateness Working Group (VAWG) engaged three clinical working groups (CWG) to consider the causes of variation in surgical practice.

Objectives Using the Model for Understanding Success in Quality Improvement (MUSIQ) framework, we conducted a qualitative study aimed at exploring VAWG's successes and barriers.

Methods We used semi-structured interviews, meetings notes, and VAWG project documents as data for understanding the functioning and context of the CWGs.

Results Our comparison of the CWGs highlights the similarities and differences between these groups. All groups identified next steps for understanding the root causes of variation; however, even in the CWG that exhibited positive micro contextual attributes and that made the most progress, success was limited due to the external environment common to all three CWGs. The external environment, the Saskatchewan health care system, did not signal that this work was a priority and physicians did not have the structural support needed to fully engage and advance this work.

Conclusions Our research highlights the importance of both micro and macro contextual factors critical to the success of physician-driven quality improvement. The MUSIQ framework identifies features of the Saskatchewan context that can be built upon, and areas were significant work and investment is needed to increase the likelihood of success in future projects. Lessons learned are impacting the implementation of subsequent work; however, there are still critical factors to be addressed in Saskatchewan. 